\title{
Fighting cancer with growing complexity
}

\author{
Robert Skopec* \\ Researcher Analyst, Department 01, AXON, Dubnik, Slovakia
}

\begin{abstract}
Cancer dogma holds that most malignancies are caused by DNA mutations inside the nuclei of cells, mutations that ultimately lead to runaway cellular proliferation. Given the millions of genetic blips that have been associated with various cancers, the illness has actually come to be seen as a complex of diseases for which personalized treatments might offer the best chances of success. But prevailing oncology orthodoxy has its detractors, and some cancer biologists feel that while mutations are nearly ubiquitous in cancer, they may not always be the driving force for disease. Cancer, they suggest, might actually be as much a disorder of altered energy production as it is genetic damage.
\end{abstract}

\section{Introduction}

As tumors grow they acquire mutations, some of which create neoantigens that influence the response of patients to immune checkpoint inhibitors. It was explored the impact of neoantigen intra-tumor heterogeneity (ITH) on anti-tumor immunity. Through integrated analysis of ITH and neoantigen burden, has been demonstrated a relationship between clonal neoantigen burden and overall survival in primary lung adenocarcinomas. CD8 ${ }^{+}$tumorinfiltrating lymphocytes reactive to clonal neoantigens were identified in early-stage non-small cell lung cancer (NSCLC) and expressed high levels of PD-1. Sensitivity to PD-1 and CTLA-4 blockade in patients with advanced NSCLC and melanoma was enhanced in tumors enriched for clonal neoantigens. T cells recognizing clonal neoantigens were detectable in patients with durable clinical benefit. Cytotoxic chemotherapy-induced subclonal neoantigens, contributing to an increased mutational load, were enriched in certain poor responders. These data suggest that neoantigen heterogeneity may influence immune surveillance and support therapeutic developments targeting clonal neoantigens [1].

Similar to how vaccines protect us from certain infections, the treatment attempts to recruit the body's own immune system to attack and destroy specific entities - in this case cancer cells. Normally, the various components of the immune system - which include white blood cells such as $\mathrm{T}$ cells - protect against cancer by killing tumor cells, although some tumors are able to evade these natural defenses.

When this occurs and cancer develops to an advanced stage, the immune system is often suppressed. Numerous factors are thought to be responsible for this effect, ranging from tumor cells' ability to damage immune cells, to a decrease in white blood cell production when cancer spreads to the bone marrow.

Vaccines typically work by injecting a patient with small amounts of antigens, substances that are capable of eliciting an immune response, which stimulate the body to produce antibodies that specialize in the labeling and destruction of that particular entity. The ability to generate these antibodies is retained for a period of time, meaning that the immune system can then fend off future cases of the same illnesses.

Accordingly, the new vaccine currently being tested is comprised of small fragments of an enzyme found in cancer cells. Called human telomerase reverse transcriptase (hTERT), this regulates the length of the protective caps on chromosomes called telomeres, enabling the cells to divide continuously. Scientists are hopeful that this will stimulate patients' immune systems to produce antibodies that can target this enzyme, thereby facilitating the destruction of cancer cells.

To give the process a kick-start, the vaccine is being combined with low doses of chemotherapy, in order to kill some of the tumor cells and disinhibit the immune system. The investigators behind the trial believe the vaccine could prove effective for all types of solid tumors, and are testing its safety and efficacy on patients whose cancer has been diagnosed as terminal [2].

\section{Fighting cancer by a diets}

Idea of diet in cancer traces back to the work of German physician Otto Warburg who, in the 1920s, reported that rather than generating energy using the oxygen-based process of respiration as healthy cells do, cancer cells prefer the anaerobic, or oxygen-free, process of fermentation. Not all products of fermentation are as welcome as beer, wine and cheese [3].

Boston College biology professor Dr. Thomas Seyfried is a leading proponent of the metabolic theory of cancer. He proselytizes Warburg's findings and in 2012 published an academic book called Cancer as a Metabolic Disease that lays out the evidence behind his beliefs (Currently the book's Facebook page has over 6,000 followers and a lively exchange of self-help tips) [4].

Seyfried argues that decades of research, including his own, support the idea that aberrant metabolism can somehow induce malignancy. Research supports at the idea that limiting the fuels

Correspondence to: Robert Skopec, Mgr., researcher-analyst, Department 01, AXON, Dubnik, Slovakia, E-mail: zxcbnvm7@gmail.com

Key words: cancer, DNA mutation, proliferation, oncology orthodoxy, personalized treatments, cancer biologists, altered energy production, fermentation, metabolic theory, self-help

Received: March 28, 2016; Accepted: May 03, 2016; Published: May 06, 2016 
available for fermentation - that is, the sugar glucose and the amino acid glutamine - is an overlooked approach to aid treatment. $\mathrm{He}$ specifically implicates mitochondria, our energy-producing organelles, in spurring on malignancy [3].

This belief is in part based on work from the '70s and '80s showing that if the cytoplasm (the buoyant cellular goo that contains the mitochondria) is transferred from a normal cell to a tumorigenic cell (one with the potential to develop into a cancer) the tendency toward cancer is suppressed. Conversely, animal research has shown that transferring the nucleus of a malignant cell into the cytoplasm of a normal cell inhibits the tumor potential of that initially malignant cell, implying that whatever is causing the cancer lies in the cytoplasm, not the nucleus [3].

\section{Cancerous cells actively recruit healthy cells into tumors}

The Soll's team discovered that cancerous cells actively recruit healthy cells into tumors by extending a cable of sorts to grab their neighbors-both cancerous and healthy-and reel them in. Moreover, the Iowa researchers report that as little as five percent of cancerous cells are needed to form the tumors, a ratio that heretofore had been unknown.

It's not like things sticking to each other, said David Soll, biology professor at the UI and corresponding author on the paper, published in the American Journal of Cancer Research. It's that these cells go out and actively recruit. It's complicated stuff, and it's not passive. No one had a clue that there were specialized cells in this process, and that it's a small number that pulls all the rest in.

It was showed that only cancerous cells (from a variety of cancers, including lung, skin, and aggressive brain tumors known as glioblastomas) engaged in tumor formation by actively soliciting other cells. Like evil-minded envoys, individual cancer cells extend themselves outward from the original cluster, probing for other cells in the area, the researchers observed. Once it detects one, the extended cell latches on and pulls it in, forming a larger mass. The cellular cables. activity continues, the cancerous extensions drawing in more and more cells-including healthy cells—as the tumor enlarges.

The question is how these cells know what to do. Soll hypothesizes they're reaching back to a primitive past, when these cells were programmed to form embryos. The cancerous cells-masquerading as embryo-forming cells-recruit other cells to make tissue that then forms the layered, self-sustaining architecture needed for a tumor to form and thrive.

Cancerous cells "recruit" cells into tumors by extending a bridge of sorts and reeling in both healthy and cancerous cells. The UI researchers documented the phenomenon for the first time in real time and in 3-D.

The results here extend our original observation that tumorigenic cell lines and fresh tumor cells possess the unique capacity to undergo coalescence through the active formation.

The finding lends more weight to the idea that tumors are created concurrently, in multiple locations, by individual clusters of cells that employ the cancer-cell cables to draw in more cells and enlarge themselves. Some have argued that tumors come about more by cellular changes within the masses, known as the cancer stem cell theory.

Soll's team also discovered that the Mo-Vi10' cells move at 92 microns per hour, about twice the speed of healthy cells. That's important because it helps scientists better understand how quickly tumors can be created [5].

\section{Shared pathology between cancer and metabolic syndrome}

We can outgo here from the existing concept of "shared pathology" between cancer and metabolic syndrome. It was found several similarities between gene signatures for cancer and gene expression signatures from inflammatory and gastrointestinal diseases. The genes common to these diseases regulate lipid metabolism and cholesterol biosynthesis. The major pathway common to both diseases is chronic inflammation, which is now considered a critical hallmark of carcinogenesis, and the molecular and biochemical mechanisms linking inflammation, lipid metabolism, and cancer. The mechanistic links between inflammation and digestive disorders are showing increasing importance for the carcinogenesis et al.

\section{The mechanistic links between inflammation and digestive disorders}

If the inflammatory microenvironment has been created in tumors, there are mechanisms which sustain it. First, the cytokines which activated the transcription factors NF- $\mathrm{kB}$ and STAT- 3 in tumor cells also activate these same transcription factors in inflammatory cells, and tumor stromal cells, which in turn results in more inflammatory mediators. The cancer-related inflammatory microenvironment is so enhanced by activation of inflammation in cells surrounding the tumor. The sustaining smoldering cancer-related inflammation has many tumor-promoting effects. The cancer-related inflammation also involves inflammation induced by reactive oxygen and nitrogen species. These free radicals damage DNA proteins, and lipids which result in gene mutation and accumulation of advanced glycation end products (AGE). Interaction of AGE with its receptor (RAGE) triggers a chronic inflammation by activation of NF- $\mathrm{BB}$, at sites of tissue damage. The activated NF- $\mathrm{BB}$, overrides endogenous antiinflammation mechanisms and leads to sustained inflammation. The importance of the RAGE-AGE interaction was proved by showing that transgenic mice lacking RAGE protein, developed skin tumors with low levels of pro-inflammatory mediators and reduced numbers of infiltrating immune cells, compared with wild type mice.

\section{Signaling pathways and transcription factors in chronic inflammation}

Our understanding of signaling pathways and transcription factors in chronic inflammation come from pre-clinical studies, and have clinical relevance since certain anti-inflammatory drugs, like nonsteroidal anti-inflammatory drugs (NSAIDs) and COX-2 specific inhibitors have proven anticancer effects in pre-clinical tumor models and clinical trials. These anti-inflammatory drugs interfere with eicosanoid signaling and metabolism, suppress the neoplastic process, and can decrease oxidative stress and angiogenesis. The potent antitumor effects of phytochemicals, such as curcumin, from turmeric, the green tea polyphenol epigallocatechin gallate (EGCG), and resveratrol from grapes, are attributed to their anti-inflammatory activities. Guggulsterone, the major active component of the gum resin from Commiphora wightii and Commiphora mukul, is used to treat internal tumors, obesity, liver disorders, and malignant sores and ulcers. Guggulsterone was shown to induce apoptotic cell death and suppress proliferation, invasion, angiogenesis and metastasis of tumor cells. All four phytochemicals (curcumin, EGCG, resveratrol and guggulsterone) 
inhibit inflammation by suppressing transcriptional activity of NF- $\kappa$ B. In addition, guggulsterone also inhibit the transcriptional activity of STAT-3.

Shared pathology between cancer and metabolic syndrome is involving a cluster of related diseases (type 2 diabetes mellitus, cardiovascular disease, and obesity) which share abnormalities like chronic inflammation, dyslipidemia, metabolism in tumors, obesity mechanisms by they lead to chronic inflammation.

\section{Tumor cells metabolize large amounts of lipids}

Tumor cells metabolize large amounts of lipids, while lipid biosynthesis and desaturation of lipids are required for tumor cell survival. Enzymes, like fatty acid synthase and stearoyl-CoA desaturase, are often overexpressed in tumor cells and are potential targets for anticancer drugs. Cholesterol serves as a precursor for the synthesis of many sex hormones has been linked to increased risk of prostate cancer. The mechanism linking cancer and cholesterol is controversial, because antineoplastic therapies influence one's lipid profile, and antihyper lipidemic drugs in turn, influence the process of malignancy.

Recent data point to a direct link between the Oxidized LDL receptor 1 (OLR-1,) and cancer. ORL- 1 act as an oncogene, by activating the NF$\kappa \mathrm{B}$, which in turn, induces expression of its target genes responsible for lipogenesis, cell proliferation, cell migration, inflammation, and inhibition of apoptosis. The oxidized LDL itself can directly promote tumors by enhancing the generation of reactive oxygen species which damage and mutate DNA. Oxidized LDL indirectly promotes tumors by inducing proinflammatory changes in macrophages and reduces their phagocytic capacity towards dying tumor cells.

Epidemiological data have linked a high body index (BMI) and obesity in both genders to an enhanced risk of colorectal, esophageal, kidney cancer, non-Hodgkin's lymphoma, and multiple myeloma. Myelomas were especially linked to obesity in males, and cancer of the breast and cervix have been linked to obesity in females. Indeed, approximately $30-50 \%$ of deaths caused by breast cancer are due to obesity.

\section{Obesity and cancers of digestive tract}

There is also an association between obesity and cancers of the digestive tract, because obesity-associated hormones, and growth factors contribute to pro-inflammatory environment created by crosstalk between epithelial tumor cells, adipocytes, and inflammatory cells. Dysregulation of cytokines like TNF- $\alpha$ and interleukin-6 (IL-6) and adipokines like lectin and adiponectin contribute to the low-grade inflammation thet is a hallmark of obesity. Lectin is mainly regulated by insulin-induced changes of adipocyte metabolism and helps to prevent weight gain, while adiponectin increase insulin sensitivity and reduce adipogenic inflammation. It means, adiponectin oppose the actions of pro-inflammatory cytokines like TNF- $\alpha$ and IL-6, and monocyte chemoattractant protein-1 (MCP-1).

The obese patients have increased concentrations of $\mathrm{C}$-reactive protein (CRP), the pro-inflammatory cytokine TNF- $\alpha$, and decreased concentrations of adiponectin. Since, levels of adiponectin did increase during weight loss, suggesting that weight loss may be able to restore an anti-inflammatory condition. Strong clinical evidence linking obesity, adipokines, and carcinogenesis and very obese patients suffering from cancer or cardiovascular events had a $30 \%$ reduction in mortality following surgery for weight loss.
Lipids like a cholesterol and oxidized LDL play a significant role in tumorigenesis, nuclear receptors are equally important. Nuclear receptors are a unique family of transcription factors which bind DNA and also bind lipid ligand. These nuclear receptors sense specific lipids and regulate the expressions of specific target genes within adipose tissue.

Liver X receptors (LXRs) are cholesterol-sensing nuclear receptors that regulate lipid metabolism and transport and also suppress inflammatory signaling in macrophages by modulating activity of NF$\kappa B$. Notably, phytosterols are agonists for LXRs and are associated with a reduced incidence of colon cancer.

One of peroxisome proliferator-activated receptors (PPARs), $\operatorname{PPAR} \gamma$ is a transcription factor regulating insulin sensitivity, adipocyte differentiation, and lipid utilization in adipocytes. To regulating gene transcription, PPAR $\gamma$ binds various lipids (fatty acids, sterols) and functions like a major sensor of lipid metabolism, and are important components of the molecular pathways interconnecting cancer development and metabolic syndrome.

Activation of farnesoid X receptor (FXR) have also anti-tumor effects. FXR deficient mice show increased susceptibility to intestinal tumorigenesis, and to inflammation induced by the endotoxin, lipopolysacharide (LPS). FXR is a specific bile acid receptor and serves as an important drug target for prevention of colorectal cancer, because elevated excretion of secondary bile acids is a strong risk factor for colorectal cancer. Interestingly, guggulsterone's efficacy against hyperlipidemia and its ability to bind FXR also make it a useful drug for colon cancer. These data suggest that normal levels of FXR expression and activity have important anti-inflammatory and anti-tumor effects.

\section{A link between type 2 diabetes mellitus and cancer}

Epidemiological data point a link between type 2 diabetes mellitus (T2DM) and cancer, which can be independent of obesity. It is becoming evidenced, that independent of obesity, T2DM can be a strong predictor of mortality from cancer of the colon, pancreas, female breast, male liver, and bladder.

The physiological link between obesity, T2DM, and cancer arises, because the adipose tissue in obese individuals produces high levels of free fatty acids, triglycerides, lectin, and pro-inflammatory cytokines. These metabolic changes increase insulin secretion and can lead to insulin resistance is common in diabetes. Obesity and elevated levels induce more secretion of insulin like growth factor 1 (IGF-1), which stimulates cell growth and proliferation.

A biochemical link between cancer and T2DM exists, because signaling through insulin receptor and insulin like growth factor 1 receptor (IGF-1R) is increased in the hyperinsulinemic condition of diabetics. The hyperactive IGF-1/IGF-1R-axis in diabetic individuals drive survival, proliferation, and growth of tumor cells. Overexpression of IGF-1R is common in several cancers, and pre-clinical studies show that downregulation of IGF-1R signals can reverse the neoplastic phenotype and sensitize cells to anticancer treatments (IGF-1R inhibitors).

Another biochemical link between cancer and T2DM arises because hyperglycemia generates oxidative stress, which in turn leads to accumulation of modified forms of DNA, protein, and lipids. The modified macromolecules can function abnormally and initiate carcinogenesis. Several products of oxidative stress, like advanced glycation end products (AGE), have pro-inflammatory effects. 
AGE, consist of glycated, carbonylated, and nitrosylated proteins, accumulates due to aging and diabetes. AGE interacts with its receptor (RAGE) and further enhances oxidative stress, induces inflammation, and significantly increasing the risk for cancers in diabetic patients.

We have explained how the shared pathology across obesity, metabolic syndrome, and cancers involves biochemical aberrations in signaling pathways regulating lipid metabolism and chronic inflammation. The diet can contribute to the pathophysiology of metabolic syndrome and cancer. Many human studies have found high levels of systemic markers of inflammation (high-sensitivity C-reactive protein: Hs-CRP), interleukin-6 (IL-6), and TNF- $\alpha$ in individuals with low-fiber, high-fat diets. The ratio of omega-6/omega-3 fatty acids showed the strongest positive correlations with increased levels of most inflammation markers, and this ratio may constitute a predictor of lowgrade chronic inflammation.

Today there are several studies on the effects of diet on inflammation markers and the risk of cancer, the influence of digestion on the risk of contracting cancer, etc. The gut microflora (influenced by diet and digestion) can influence the pathways linking diet and low-grade inflammation. For example, the fat depots from mice with colitis showed increased expression of inflammatory cytokines and the nuclear-receptors PPAR $\gamma$ and FXR.

Administration of probiotics reversed these pro-inflammatory effects and normalized the gut microflora. The normal gut microflora have important anti-inflammatory effects. Also PPAR $\gamma$, LXR, FXR are important components of molecular defense mechanism to protect against accumulation of toxic endogenous lipids and bile acids_which accumulate in diet induced hyperlipidemia. The drugs modulating FXR activity and guggulsterone have a role in treatment of colon cancer, associated with fatty diets and elevated secretion of bile.

\section{Mitochondrial dysfunction as the primary driver of cancer}

More recent research shows that the introduction of mutations in mitochondrial DNA (former single-celled organisms that our cells eventually engulfed, mitochondria have their own genetic material) reduces the tumor protection purportedly provided by normal mitochondria.

"If you look at the data, you could say that there is clear evidence that cancer is a genetic disease since we can inherit mutations associated with increased cancer risk," says Seyfried, "but many of these mutations disturb cellular respiration. And many non-inherited causes of cancer like radiation impair mitochondrial function." Seyfried's colleague Dominic D'Agostino, a biology professor at the University of South Florida also subscribes to the idea that the primary driver of cancer is mitochondrial dysfunction, which can be induced by any number of carcinogens - genetic predilections, radiation, chemical exposures and diet among them [3].

Not only do many mutations and pathways associated with cancer impair mitochondrial function and cell metabolism, he says, but injured mitochondria also produce volatile compounds called "reactive oxygen species" that can damage DNA. "This can explain why most cancers have mutations," he speculates, "in many cases they're secondary to mitochondrial damage" [3].

Dr. E. Aubrey Thompson, a cancer biologist at the Mayo Clinic who in his own words is "strongly on the mutation side," acknowledges that cancer cells re-orchestrate their metabolic activities and that interfering with cancer metabolism is a potentially fruitful area of research. "There are hundreds of labs already working on this right now," he says. Yet, he adds genially, "there is no evidence of malignancy developing in the absence of mutations. Anyone who thinks otherwise is obligated to design an experiment to disprove this concept ... that's how science works" [3].

It turns out there is some evidence that this might happen, but it's limited. Seyfried pointed me to a 2015 paper by Dr. Stuart Baker of the National Cancer Institute that reviews four recent studies reporting numerous tumors in which zero mutations were found. Seyfried acknowledges that mutations might have been found with more thorough screening and better DNA sequencing technology [4].

Memorial Sloan-Kettering Cancer Center President and CEO Dr. Craig Thompson (not to be confused with Mayo's Thompson) hedges on the metabolic theory of cancer. "While the arguments raised [by Seyfried and others] have been considered by the growing field of cancer metabolism, most investigators have moved on to consider other explanations for the observations," he said in an interview. Still, he has recently written in support of some aspects of the theory with therapeutic implications.

In a way, the various competing cancer theories aren't completely at odds. "Despite all the talk of controversy in the field, the concepts that [Seyfried] and his colleagues are advancing are not really that novel," says Mayo's Thompson, "I think everybody who works in cancer biology today appreciates the fact that there are many different processes involved in a conversion of a normal cell to a tumor cell." $\mathrm{He}$ believes a coalescence of pernicious influences is required for a cancer to develop. "One of these processes is probably altered metabolic activity," he says. "But cancers also must acquire mutations, change the way they interact with neighboring cells and learn to evade the immune system. Every single one of these processes is probably essential to cancer development" [3].

Matthew Vander Heiden, a biologist at MIT and oncologist at the Dana Farber Cancer Institute, also says many factors are necessary to induce cancer, including what could be considered on the other major theory of the origin of malignancies, that they result from the impairment of signaling pathways that control cell division. "My guess is it's probably metabolic, and it's probably genetic and it's probably cell signaling. I'm not sure you can separate these out since they all appear to be so interrelated," he explains. Regardless of the initial cancer trigger, the ultimate end of the biomedical bickering is to help patients. And as Vander Heiden points out, not only are researchers and pharmaceutical companies already developing drugs that target metabolic pathways, such drugs have been around for some time. "I think targeting metabolic pathways in cancer is a great idea. We already have five or six mainstay chemotherapies that yes, attack cell division machinery, but also target metabolism. They're just not billed that way. Seyfried is skeptical that medicines alone will cure cancer. Instead he and many of his colleagues - including Dr. Eugene Fine from the Albert Einstein College of Medicine and University of Pittsburgh neurosurgeon Dr. Joseph Maroon - are focusing on the potential of dietary approaches to contain the disease. There's particular interest in the ketogenic diet, similar to the low-carb Atkins diet that is low in sugar and high in fat. It's intended to starve cancer cells of the glucose they use for fermentation [3].

\section{No reason to think patients must be poisoned to be healthy}

The drugs we have now are so toxic and there's no reason people 
should have to be poisoned to be healthy. There are a number of studies, including those we've published, showing a direct relationship between the ketogenic diet and slowed tumor growth," says Seyfried, also citing the work of Dr. Valter Longo at University of California, Davis showing that low- calorie diets are linked with slowed tumor growth and improved response to chemotherapy. Why spend all this money going after all these different pathways involved in cancer when you can simply go after the key fuels? [4]

\section{Exercise protects against cancer}

AMPLE evidence shows that exercising regularly reduces the risk of cancer. Similarly, those who have survived the disease are less likely to see it return if they engage in lots of physical activity after treatment. All this suggests that such activity triggers a reaction in the body which somehow thwarts cancer cells, but the details of the process have remained murky. Now, a team led by Pernille Hojman at Copenhagen University Hospital, in Denmark, has reported in Cell Metabolism that the key to the mystery is adrenalin [6].

She began her work by verifying that exercise truly does have beneficial anti-tumour effects. She and her colleagues gave some of the mice in their laboratory activity wheels, which the animals could run around inside as much as they liked. Other mice, meanwhile, were given no opportunity to exercise beyond moving about inside their cages. The researchers then induced mice of both sorts to develop one of three types of cancer. Some, they injected with a substance called diethylnitrosamine, which causes liver cancer. Others, they injected below the skin with melanoma cells, which then set up shop where they had been injected. Others still had their tails inoculated with melanoma cells. In mice, previous experience has shown, this leads to melanomas forming in the lungs.

The results were instructive. While all mice injected under the skin with melanoma cells developed that cancer, the tumors in animals which had had access to a running wheel were $61 \%$ smaller after six weeks than were those in mice that had been unable to exercise. A similar reduction in size (58\%) pertained to lung tumor. And, of the mice injected with diethylnitrosamine, only $31 \%$ of those with wheels in their enclosures developed tumors at all-in contrast to a $75 \%$ tumour-development rate in mice lacking access to a wheel.

To try to understand why exercise does this, Dr Hojman and her team put under a microscope some of the tumours they had induced. They found that those from well-exercised mice contained more immune cells than equivalent tumors from inactive animals. Specifically, the former had double the number of cytotoxic T-cells, which kill off body cells that are damaged, malfunctioning or infected with viruses. They also had five times more natural killer cells, a type that sounds the alarm and attracts other immune cells.

To find out, she ran a fourth experiment, in which mice induced to have cancer were injected either with epinephrine or with saline. The hormone performed well, reducing the growth of tumours by $61 \%$ in mice that had no access to a wheel. However, this was not as impressive as the reduction of $74 \%$ which the team saw in control mice that got regular exercise. There was, they concluded, something else involved. And they found it in the form of interleukin-6.

Levels of this molecule also spike during exercise-and it, too, helps immune cells home in on tumors. When Dr Hojman and her colleagues exposed sedentary mice both to epinephrine and to interleukin-6, the rodents' immune systems attacked the tumors in their bodies as effectively as if those animals had engaged in regular wheel-runs.
Above findings, then, suggest that epinephrine and interleukin-6 could be used as anti-tumor drugs. They are not proposing that they should be a substitute for exercise in those who are merely lazy-not least because exercise brings benefits beyond curbing oncogenesis. But people who are too old or too ill to be active might thus gain exercise's anticancer benefits without the need to get sweaty [6].

\section{Diet and nutrient-based cancer treatments}

The idea of fighting cancer by changing what patients eat has obvious appeal, but it also raises worries. "I get a little scared when people start talking about diet for cancer since you can quickly get into pseudoscience here," Mayo's Thompson counters. He points out that data supporting the ketogenic diet in cancer are limited - and further that rigorous dietary studies are incredibly hard to pull off. "The drug companies aren't going to fund these types of trials," he says. "They can't make money marketing a diet" [3].

Vander Heiden is also wary of many dietary claims, in part because of biased expectations. "It seems that people have often decided what diet they think is best before they do a study," he says. "There's a difference between setting out to prove something is a good therapy and asking what therapy is best" [7].

His own work has shown that certain dietary interventions can be more effective than drugs at treating cancer in mice, but he says panacean claims about the ketogenic diet specifically are a bit premature. "I think it's a really interesting hypothesis that should continue to be tested, but to claim that cancer is all metabolic or all genetic is probably incorrect," he says. Usually in science when you have something as complex as cancer, ascribing it to one particular cause is often going to far" $[8,9]$.

Even Seyfried acknowledges, despite his zeal for treating cancer by tinkering with calories, that in all likelihood diet and nutrient-based cancer treatments will serve as adjuncts to existing therapies. But what would be wrong with that? "We're slowing the tumor down and making it extremely vulnerable to lower, less-toxic doses of available drugs," he says, "When people are locked into an ideology created by a dogma they tend not to focus on rational alternatives."

\section{The immune response is directly linked to brain func- tioning}

We can improve our immunity by maintaining brain health Psyconeuroimmunology is the field that combines study of endocrine, neurological and immune functions. Problem is it's not mainstream. Mainstream endocrinologists, neurologists, immunologists mine their respective streams in strict silos. Psychoneuroimmunology thus remains a fringe field, its results often greeted with skepticism and disdain by the standard bearers of these fields [10].

Mosaic science recently extensively reported on one of the most famous studies mainstream immunologists hardly ever discuss [11]. In 1975, Robert Ader, a physiologist at the University of Rochester, New York, gave rats saccharin to drink. He conditioned some of them to associate the sweet saccharin taste with an aversive experience by simultaneously infecting them with Cyclophosphamide to make them feel sick. When sugar water + cyclophosphamide conditioned rats were offered sugar water alone, they refused to drink it. So Ader then forcefed it to them using an eyedropper. All the rats died. To understand what happened, Ader compared immune responses of aversion-conditioned rats to placebo-treated ones and found circulating antibodies of the former were dramatically lower [12]. He concluded these rats had become immunosuppressed. While that interpretation was an error, 
simply because cyclophosphamide gets rid of B cells, the source of antibodies, that still doesn't explain why aversion-conditioned rats should die from drinking innocuous sugar water [13].

More recently, Kevin J. Tracey showed that rats that should have died from septic shock from lethal endotoxin injection didn't simply because their vagus nerve was simultaneously stimulated [14].

Simply remarkable that parasympathetic pathway stimulation at the right moment was all it took to avert an irreversible and precipitous fatal collapse [15].

These observations lead us directly to the Placebo effect. Far from being inert/neutral, the placebo experience induces tangible physiological effects not just in conditions where brain involvement is well known but also in others where it isn't. Clearly, the brain is involved. Fabrizio Benedetti, a prominent placebo researcher even goes so far as to say that, 'words and drugs may use the very same mechanism and the very same biochemical pathways' [16].

Problem with seeking a prescription for how to use brain health to improve immune health is best described by social psychologist Richard E. Nisbett. In a nutshell, Nisbett says we are largely unaware of our motivations, why we do what we do, because we are unaware of our cognitive process [17].

The single thing I've done that has gotten the most notice was my work with Tim Wilson showing that we have very little access to the cognitive processes that go on that produce our behavior. We are constantly being influenced by things that we don't recognize have had an influence, and which are sometimes embarrassing to know. That isn't why we're unaware of them. We're unaware of them because we don't have access to our cognitive process. We claim that we do. You ask me why I do something, I'll give you an answer, although you'll probably believe it more than I will because I'm so aware of the extent to which we're unaware of what goes on [18].

This means if we're largely and often unaware of what we're unaware of, deliberation can only do so much to improve brain health. Of course, on the other side of the coin are practitioners of techniques such as Transcendental Meditation and other types of mindfulness meditation such as Matthieu Ricard who would disagree. Perhaps they have a point, that with training we can improve our awareness of the sub-conscious. At least one pilot study shows that an 8 -week meditation course improved circulating anti-influenza vaccine antibody titers even when tested 4 months later. At that point volunteers were doing only 15-minute meditation sessions just 2 or 3 times a week [19], and yet it made a difference.

\section{References}

1. McGranahan N, Furness AJ, Rosenthal R, Ramskov S, Lyngaa R, et al. (2016) Clonal neoantigens elicit $\mathrm{T}$ cell immunoreactivity and sensitivity to immune checkpoint blockade. Science 351: 1463-1469.

2. Taub B (2016) Human trials for a radical new cancer vaccine just began. IFL Science

3. http://www.npr.org/sections/health-shots/2016/03/05/468285545/fighting-cancer-byputting-tumor-cells-on-a-diet

4. Seyfried T (2012) Cancer as a Metabolic Disease. John Wiley \& Sons, Inc. USA

5. Soll D et al., (2016) Cancer riddle, solved: Researchers reveal how cancer cells form tumors. American Journal of Cancer Research.

6. http://www.economist.com/news/science-and-technology/21693419-active-lifeprotects-against-cancer-researchers-now-understand-why-run-day

7. Kracht MJ, Zaldumbide A, Roep BO (2016) Neoantigens and Microenvironment in Type 1 Diabetes: Lessons from Antitumor Immunity. Trends Endocrinol metab. [Crossref]

8. Skopec R (2015) Intelligent Evolution, Complexity and Self-Organization. Neuro Quantology 3: 299-303.

9. Skopec R (2016) Translational Biomedicine and Dichotomous Correlations of Masking. Translational Biomedicine 7: 47

10. Skopec R (2016) Allergy as precursor of carcinogenesis. Glob Vaccines Immunol 1: 19-23.

11. http://mosaicscience.com/story/medicine-without-the-medicine-how-to-train-yourimmune-system-placebo

12. Ader R, Cohen N (1975) Behaviorally conditioned immunosuppression. Psychosom Med 37: 333-340. [Crossref]

13. Hsu CC, Tseng LM, Lee HC (Crossref) Role of mitochondrial dysfunction in cancer progression Exp Biol Med (Maywood). [Crossref]

14. Borovikova LV1, Ivanova S, Zhang M, Yang H, Botchkina GI, et al. (2000) Vagus nerve stimulation attenuates the systemic inflammatory response to endotoxin. Nature 405: 458-462. [Crossref]

15. Cardenia V, Rodriguez-Estrada MT, Lorenzini A, Bandini E, Angeloni C, et al. (2016) Effect of broccoli extract enriched diet on liver cholesterol oxidation in rats subjected to exhaustive exercise. J Steroid Biochem Mol Biol. [Crossref]

16. Benedetti F (2012) The placebo response: science versus ethics and the vulnerability of the patient. World Psychiatry 11: 70-72. [Crossref]

17. https://www.edge.org/conversation/richard_nisbett-the-crusade-against-multipleregression-analysis

18. Skopec R (2016) Mutagenesis Associated with DNA-Damage-Stress Response. Journal of Analytical Oncology 2016: 33-37.

19. Davidson RJ, Kabat-Zinn J, Schumacher J, Rosenkranz M, Muller D, et al. (2003) Alterations in brain and immune function produced by mindfulness meditation. Psychosomatic Medicine 65: 564-570. [Crossref]

Copyright: (C2016 Skopec R. This is an open-access article distributed under the terms of the Creative Commons Attribution License, which permits unrestricted use, distribution, and reproduction in any medium, provided the original author and source are credited. 